\title{
18.1: Electronic Compensation for Fringe-Field Effects in VAN LCOS Microdisplays
}

\author{
Dieter Cuypers \\ IMEC vzw, CMST, 9052 Zwijnaarde, Belgium
}

\author{
Herbert De Smet ${ }^{*}$, André Van Calster ${ }^{*}$ \\ Ghent University, ELIS Department, CMST, 9052 \\ Zwijnaarde, Belgium
}

\begin{abstract}
Although fringe field effects in LCOS projection panels can be suppressed sufficiently not to interfere with the optical performance needed for consumer applications, precise greylevel reproduction in professional applications still requires a detailed knowledge of the occurrence of disclinations at all driving voltages and neighboring pixel configurations. Such a study is presented in this paper, allowing the implementation of an electronic compensation for the fringe field effects.
\end{abstract}

\section{Introduction}

LCOS devices in projection offer a wealth of opportunities for high end applications. Currently available lightvalves based on the vertically aligned LC mode offer high performance on most aspects such as response speed, contrast ratio and light throughput. Digital mirror devices still outnumber LCOS devices today, but the trend towards ever higher resolution (e.g. the $2 \mathrm{k} / 4 \mathrm{k}$ devices proposed by the Digital Cinema Initiative [1]) may soon change this. An important feature of LCOS devices in this respect, are the relatively few technological hurdles for increasing resolution, making it an ideal candidate for these challenging applications.

A point of concern for liquid crystal devices with small pixels is the influence of electrical fringe fields on the optical performance. Adverse conditions may result in contouring problems, trailing edges and other highly obvious optical artifacts, as documented in e.g. [2,3]. The problem, as it occurs in vertically aligned cells is illustrated in figure 1 . The electrical field that exists between a pixel turned on and its neighbor at a different voltage causes part of the liquid crystal molecules to adopt a tilt angle opposite to the one in the main part of the pixel. This reverse tilt zone and especially the changeover from one tilt direction to the other causes changes in the optical throughput of the pixel, typically a splitting in two bright zones with a dark line in between.

A study of the parameters involved in the creation of the reverse tilt zone and its optical artifacts in the case of moderate pixel sizes has already been performed, see [4]. It is demonstrated that a well chosen pretilt angle can suppress the drawbacks of the reverse tilt zones if the ratio of pixel width to the liquid crystal layer thickness is relatively high.

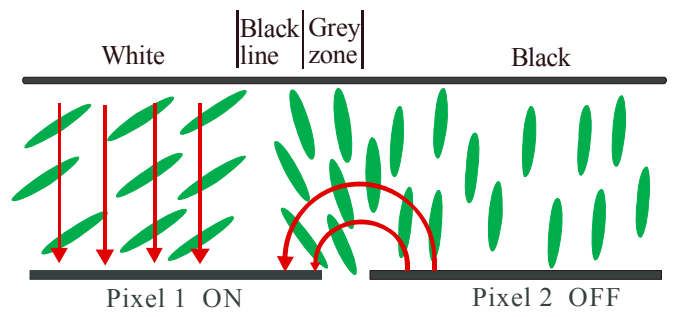

Figure 1. Illustration of the reverse tilt effect in a vertically

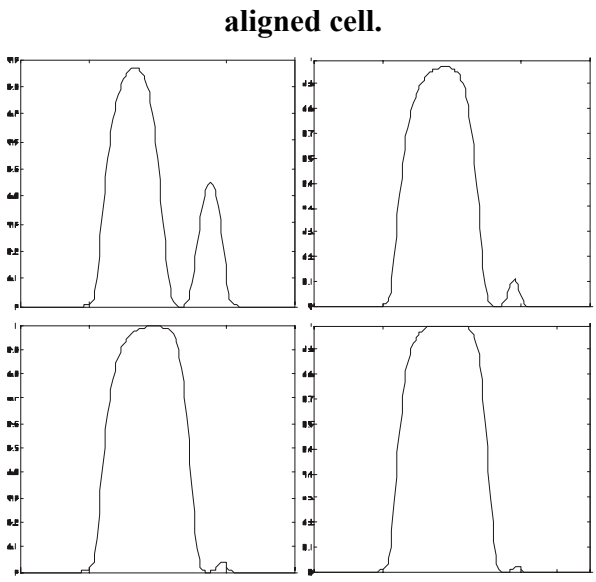

Figure 2. Influence of pretilt angle on the creation of reverse tilt zones in regular devices. Shown are calculated reflectance profiles for a single full bright pixel (located between the tick marks) surrounded by black pixels. Pixel pitch $11.5 \mu \mathrm{m}$, cell gap $3 \mu \mathrm{m}, 3 \mathrm{~V}$ driving voltage, MLC-6610. Pretilt angle is $89.5^{\circ}, 88.5^{\circ}, 87^{\circ}$ and $85^{\circ}$, from left to right and top to bottom.

This is shown in figure 2 , where the simulated optical reflectance profiles of a single full white pixel surrounded by black pixels are plotted for different pretilt angles. As a result, the fringe field effects can be controlled to the extent needed for most consumer applications. This means severe errors as described above are eliminated and the panels exhibit negligible fringe field distortions.

\section{Devices with High Pixel Density}

For high end applications however, this way of fringe field effect control may prove to be not adequate enough, for the reasons listed below.

First of all, due to the very high resolutions that must be squeezed onto an area of silicon that is still comparable to lower resolution devices, the size of the pixels shrinks at a faster rate than the thickness of the liquid crystal layer, which is reduced at a rate determined only by advancements in the liquid crystal material development. As a result the ratio of pixel pitch to cell gap becomes quite low and under such conditions the occurrence of a reverse tilt zone becomes unavoidable, regardless of the chosen pretilt angle. This does not imply that this configuration is unusable; since the resolution is so high, the changes in brightness of a single pixel on the projected image are normally not resolved by the eye anymore.

Secondly, high-end devices also require a higher fidelity in color reproduction, i.e. the grey values produced by each pixel on the lightvalve must closely match the intended ones, irrespective of external conditions like the state of surrounding pixels.

This means that although the existence of the reverse tilt zone 
may not be resolved by the eye, it does change the amount of light reflected by the pixel as compared to the originally intended value and must thus be corrected for. Similarly, if the surrounding pixels are at different voltages, this will probably affect the fringe fields and thus the strength of the reverse tilt and the resulting reflectance of the considered pixel. To keep the exact intended grey value on the screen under these varying circumstances, a correction will have to be applied.

In order to obtain the correction factors for the image data so that the reproduced image displays the exact grey levels, twodimensional simulations of the liquid crystal behavior are used to explore the influence of different neighbor configurations on the pixel reflectance. This knowledge should then allow us to pre-process the image data by correcting the individual pixel voltages.

\section{Simulation}

\subsection{Setup}

For the simulation of the liquid crystal behavior a vector model is used [4]. The considered topology for the simulation is a row of pixels, of which the middle one is taken to be brightest (highest voltage). The side pixels are kept at the same (but variable) voltage. This configuration can capture all possibly interesting configurations without introducing redundancy in the simulations. The pixel width is 8 micrometer, with an interpixel gap of $0.35 \mu \mathrm{m}$ while the cell gap is set at $2.4 \mu \mathrm{m}$. The liquid crystal properties are $\Delta \mathrm{n}=0.96, \Delta \varepsilon=-3.1, \mathrm{~K}_{11}=14.6 \mathrm{pN}, \mathrm{K}_{22}=$ $12 \mathrm{pN}$ and $\mathrm{K}_{33}=16.5 \mathrm{pN}$. Pretilt angle is fixed at $85^{\circ}$ from horizontal, oriented from left to right.

The optical profiles shown are calculated from the director profile using a simple Jones matrix method and are thus for perpendicular incident light. This accounts for the small discrepancies between the simulated profiles and the photographs shown. If obliquely incident light is taken into account using the extended Jones matrix calculation, a closer match is obtained. However, in a first approximation the 'perpendicular incidence only' approach suffices and saves computing time.

\subsection{Results}

Figure 3 shows the simulated reflectance profile for a pixel at full brightness under the above described conditions. As a comparison, figure $4 \mathrm{a}$ is a photograph through a microscope of pixels in this configuration on an actual lightvalve. As stated earlier, the effects of the reverse tilt zone cannot be suppressed anymore for high ratios of cell gap to pixel width and the black line with a secondary bump in the pixel brightness are clearly visible.

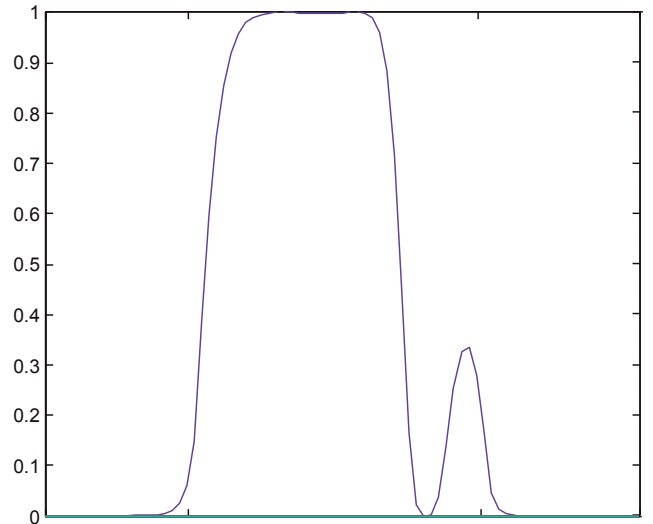

Figure 3. Simulated optical reflectance profile of a single full bright pixel neighbored by black pixels. Driven pixel is between the tick marks. See text for detailed simulation conditions.
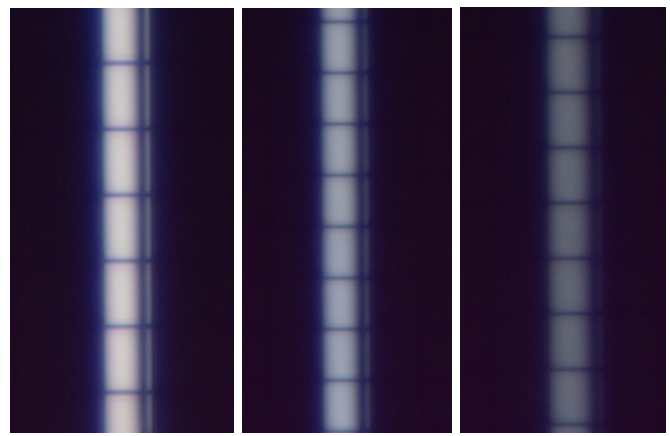

Figure 4. Micrographs of the reverse tilt zone occurring at the interface of a single line of pixels driven at $100 \%, 85 \%$ and $30 \%$ brightness neighbored by full black pixels. Pixel width $8 \mu \mathrm{m}$, cell gap $2.4 \mu \mathrm{m}$.

This general behavior is maintained if the driving voltage of middle pixel is reduced, although the shape of the main reflectance bump and the size of the secondary bump are gradually changing. This is visualized in figure 5, giving an overview of reflectance profiles for a number of brightness levels. Figure 4 (b and c) can again serve as reference, showing the excellent match between simulation and reality.

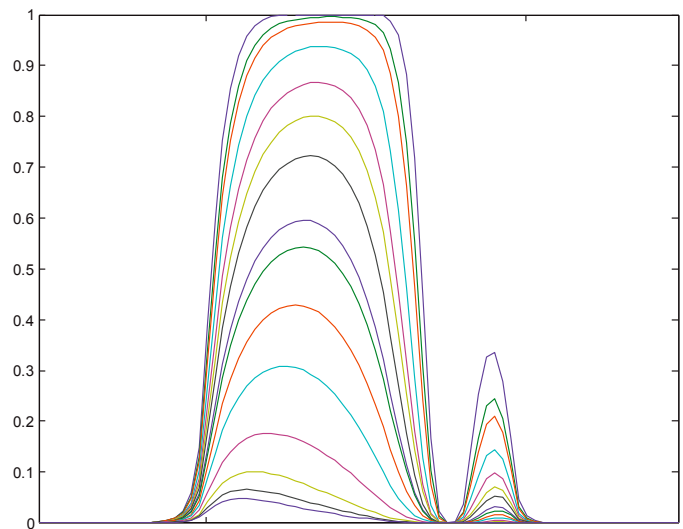

Figure 5. Optical reflectance profiles of a single pixel at several driving voltages neighbored by black pixels. Driven pixel is between the tick marks. 
It is clear that the brightness reflected by the pixel is influenced by the reverse tilt zone. If the effective reflectance for each brightness level is calculated by integrating the profile and compared to the original intended value, represented by a rectangular reflectance profile, figure 6 is obtained. The deviation from the intended brightness value increases, approximately linearly, as the brightness level drops. This illustrates that a correction for fringe field effects is more than a constant factor, but should be adapted to the brightness level.

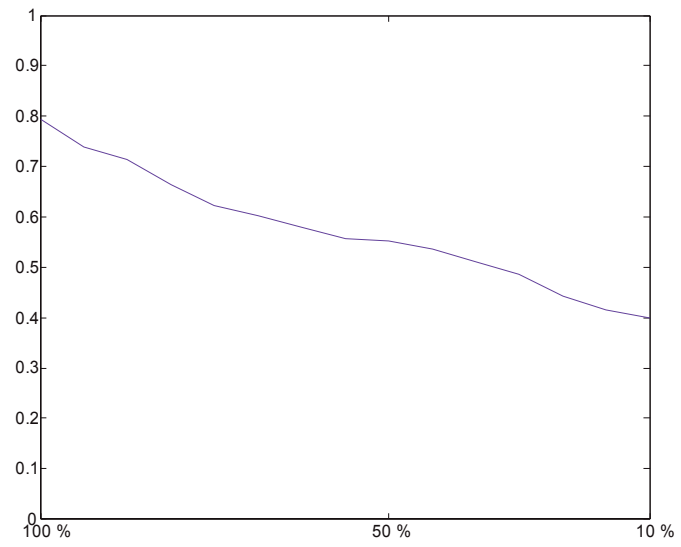

Figure 6. Ratio of the actual total reflectance of a single pixel to the intended reflectance as a function of the brightness level.

The above described exercise can of course be repeated for other brightness values for the surrounding pixels. Figure 7 shows the simulated profiles for a $100 \%$ to $55 \%$ brightness pixel surrounded by $50 \%$ brightness neighbors. It is immediately apparent that the secondary bump has disappeared. There is still a dimple in the reflectance profile where the black line used to be, but it is far less pronounced, especially when the difference between the two brightness levels is small. At the left side of the profile, the brightness of the middle pixel extends a little into the neighboring pixel. A similar set of profiles is obtained with neighboring pixels at $13 \%$ brightness, see figure 8 . Figure 9 shows this behavior reproduced on real cells.

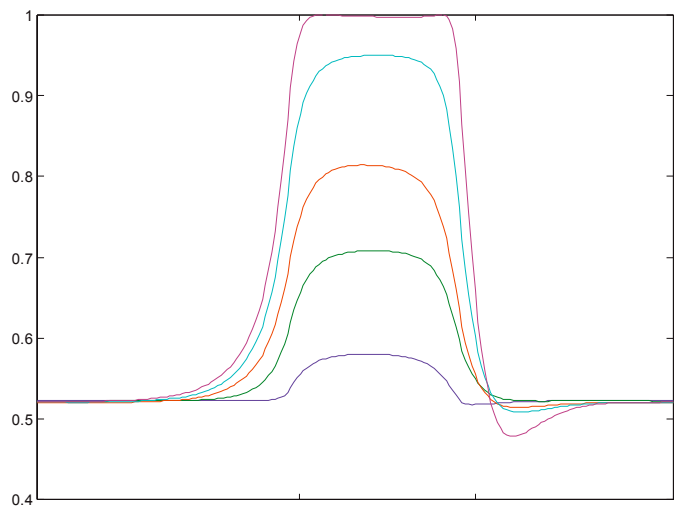

Figure 7. Optical reflectance profiles of a single pixel at several driving voltages neighbored by pixels at $50 \%$ brightness. Driven pixel is between the tick marks.

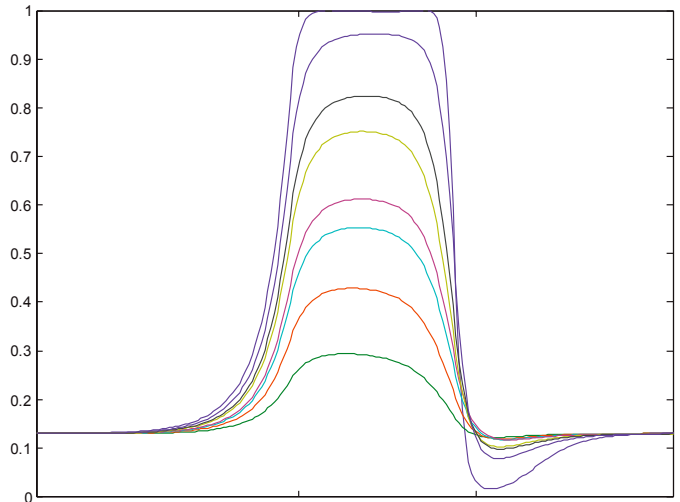

Figure 8. Optical reflectance profiles of a single pixel at several driving voltages neighbored by pixels at $13 \%$ brightness. Driven pixel is between the tick marks.

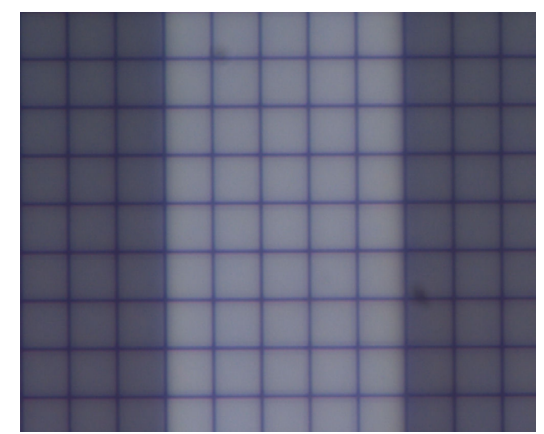

Figure 9. Micrograph of the interface of pixels at $20 \%$ brightness (sides) and $50 \%$ brightness (middle). Pixel width $8 \mu \mathrm{m}$, cell gap $2.4 \mu \mathrm{m}$.

The suppression of the secondary bump in the case of neighboring pixels at higher brightness values can be understood by realizing that the fringe field strength is substantially less in this case. Depending on the actual liquid crystal properties, the changeover can occur anywhere on the electro-optical response curve. Figures 10 and 11 show that in the case at hand, the changeover occurs very close to full black: at $2.5 \%$ brightness there is only a black line, while at $1.5 \%$ there is the onset of the secondary bump.

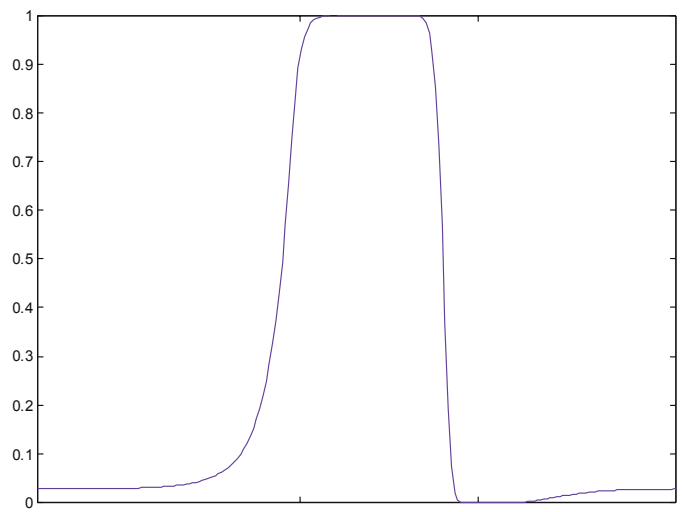

Figure 10. Optical reflectance profiles of a single full bright pixel neighbored by pixels at $2.5 \%$ brightness. Driven pixel is between the tick marks. 


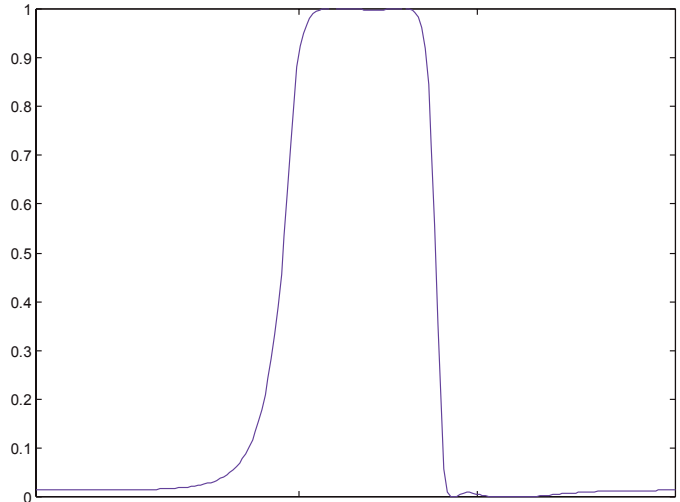

Figure 11. Optical reflectance profiles of a single full bright pixel neighbored by pixels at $1.5 \%$ brightness. Driven pixel is between the tick marks.

Again, and although the discrepancies are markedly smaller than previously, it is clear that the obtained brightness levels will usually not be the intended ones and a brightness level dependent correction must be applied.

\section{Correction Scheme}

With the simulation data, a complete matrix of deviations and thus correction factors between actual and intended brightness values can be calculated for all possible neighboring conditions.

A first, obvious correction scheme then exists of a lookup table with the possible transitions and their correction factor. Corrections may then be applied by scanning the image data, e.g. first row by row and then again column wise, correcting for the transition with pixels to the right and below. This is feasible because one can take advantage of the fact that the reverse tilt zone will always appear at the same sides of the pixel, determined only by the pretilt direction (see figure 12 for an illustration of this). Figure 12 also brings out the weak point in this scheme: the fact that the reverse tilt zone effects to the right and below the pixel are entirely independent from each other is an approximation. However, it is a reasonable approximation, with only the corner effect that is missed.

Although more elaborate correction schemes, giving higher accuracy are certainly possible, the simple lookup table system has the advantage of being very fast and cost-effective, requiring only some memory and some very basic processing power, while delivering an adequate image improvement.

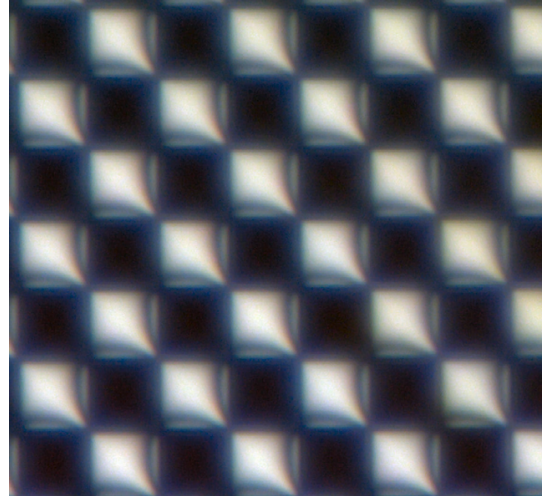

Figure 12. Micrograph of a checkerboard pixel on pixel off pattern showing the two-dimensional structure of the reverse tilt zone. Pixel width $8 \mu \mathrm{m}$, cell gap $2.4 \mu \mathrm{m}$.

\section{Conclusion}

The effects of the reverse tilt zones due to electrical fringe fields in high resolution LCOS devices have been explored and quantified by using two-dimensional simulations and comparing them with observations of real lightvalves. From these simulation results, a means of pre-correcting the image data for the deviations introduced by the fringe field effects is derived, allowing an effective use of these lightvalves in high-end applications.

\section{References}

[1] C. Colpaert, "Digital Cinema: ready for full deployment", Proceedings of the 13th International Display Workshops, pp. 1951-1954, (2006).

[2] J. Peng, R. Chang, G. Chang, J. Chiu, "Disclinations lines in VA mode LCOS displays", Proceedings of IDMC05, pp. 233-235, (2005).

[3] D. Cuypers, H. De Smet, A. Van Calster, "Fringe-field Induced Disclinations in VAN LCOS Panels", Proceedings of the 11th International Display Workshops, pp. 15411544, (2004).

[4] D. Cuypers, H. De Smet, A. Van Calster, "Fringe Field Effects in Microdisplays", SID05 Digest of Technical Papers, pp.1289-1301, (2005). 